\title{
Preface to 54-3
}

\author{
Axel Ruhe
}

Published online: 12 September 2014

(C) Springer Science+Business Media Dordrecht 2014

\section{Introduction to the contents of BIT 54:3}

Welcome to another regular issue of BIT Numerical Mathematics. These papers have been available online since more than half a year, and we get new contributions ready all the time.

These are the papers:

Andreas Asheim describes how to handle multidimensional quadrature for oscillating functions. A change to n-spherical coordinates reduces the problem to a nested univariate integration, and makes a steepest descent procedure possible. The technique is illustrated on an acoustics problem, and the application to general scattering problems is discussed.

Minghua Chen, YantaoWang, and Xiao Cheng study iterative methods for linear systems coming from numerical integration of ODEs and time-dependent PDEs by implicit Runge-Kutta and boundary value methods. They use a preconditioning strategy based on a Kronecker product splitting of the coefficient matrix.

Weihua Deng describes a second-order locally one dimensional multigrid method for a multidimensional Riesz fractional diffusion equation. This second order scheme is unconditionally stable and needs only marginally more work than a first order method.

Per Christian Hansen, James G. Nagy, and Konstantinos Tigkos describe how to handle images blurred by a rotation along an arbitrary axis. They use sparse matrix techniques to develop a fast iterative algorithm. A robust stopping rule is crucial for the usefulness of the algorithm.

\footnotetext{
A. Ruhe ( $ه)$

Numerical analysis group, Department of Mathematics, KTH, Stockholm, Sweden e-mail: ruhe@csc.kth.se
} 
Eskil Hansen and Tony Stillfjord analyze the convergence of discretizations of nonlinear parabolic equations with delay. They show that a Lie splitting gives better accuracy than implicit Euler. The technique is illustrated on a problem coming from population dynamics.

Miklós Hoffmann, Imre Juhász, and Gyula Károlyi describe a control point based curve, generalizing splines and trigonometric splines. Affine invariance, convex hull property, endpoint interpolation and global control is established.

Rong Huang, Jianzhou Liu, and Li Zhu establish a strong component wise perturbation bound for the solution of a diagonally dominant tridiagonal linear system. It is independent of the traditional condition number of the coefficient matrix, and shows how to solve such a system to high relative accuracy.

Emil Kieri solves time dependent Schrödinger equations with non-smooth potentials. He shows how to improve the convergence rate of Fourier collocation from second to fourth order. The technique is teated on an application describing higher harmonic generation from an atomic gas exposed to a laser beam

Mohamed Lamnii, Azzeddine Mazroui, and Ahmed Tijini describe how to raise the approximation order of multivariate quasi-interpolants using a blossoming approach.

Jorge Eduardo Macías-Díaz develops an exact finite-difference methodology to approximate the solution of a diffusive partial differential equation with Burgers advection and Huxley reaction law. This equation has solitary-wave solutions which are positive, bounded, and both spatially and temporally monotone. The finite difference approximation is designed to keep these properties.

Yuto Miyatake develops an energy-preserving exponentially-fitted continuous stage Runge-Kutta method for Hamiltonian systems.

Christian Perret and Wesley Petersen solve an Itô stochastic differential equation with a split step method, built up by trapezoidal steps combined with martingales, giving a two-stage method with partial overlap between successive time steps.

Magnus Svärd shows that a summation-by-parts finite difference scheme for a linear initial-boundary-value problem is stable in the maximum norm. This gives optimal convergence.

Shu-Lin Wu and Mohammad D. Al-Khaleel study semi-discrete Schwarz waveform relaxation algorithms for time dependent reaction diffusion equations. They investigate in which cases the continuous analysis is applicable and when semi discretization introduces additional errors.

That was all! I wish you all a rewarding read,

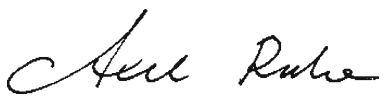

Axel Ruhe 